\title{
Bardet-Biedl Syndrome
}

National Cancer Institute

\section{Source}

National Cancer Institute. Bardet-Biedl Syndrome. NCI Thesaurus. Code C118632.

An autosomal recessive inherited syndrome caused by mutations in at least fourteen different genes, called BBS genes. It is characterized by loss of vision, obesity, diabetes, hypertension, hypercholesterolemia, polydactyly, intellectual disability, genital organs abnormalities, and delayed development of motor skills. 